\title{
MAXIMAL TRANSFERENCE AND SUMMABILITY OF MULTILINEAR FOURIER SERIES
}

\author{
LOUKAS GRAFAKOS ${ }^{\unlhd}$ and PETR HONZÍK
}

(Received 22 January 2004; revised 10 November 2004)

Communicated by A. H. Dooley

\begin{abstract}
We obtain a maximal transference theorem that relates almost everywhere convergence of multilinear Fourier series to boundedness of maximal multilinear operators. We use this and other recent results on transference and multilinear operators to deduce $L^{p}$ and almost everywhere summability of certain $m$ linear Fourier series. We formulate conditions for the convergence of multilinear series and we investigate certain kinds of summation.
\end{abstract}

2000 Mathematics subject classification: primary 42B 15, 42B25.

\section{Introduction}

Transference is a powerful tool that reveals equivalent and often unexpected reformulations of certain estimates. The study of transference of boundedness of linear operators has been pursued by several authors; for brevity we only mention the pioneering work of de Leeuw [5] that was beautifully placed into a framework of a general theory by Coifman and Weiss [4].

As an application of transference and some basic functional analysis, the $L^{p}$ convergence of Fourier series of $L^{p}$ functions on the circle $\mathbb{T}$ is equivalent to the $L^{p}$ boundedness of the Hilbert transform $H$ on $\mathbb{R}$. Likewise, the almost everywhere convergence of the Fourier series of a function on $\mathbb{T}$ whose $p^{\text {th }}$ power is integrable

The first author is supported by the National Science Foundation under grant DMS 0099881. The second author's research was supported by 201/03/0931/Grant Agency of the Czech Republic.

(c) 2006 Australian Mathematical Society 1446-7887/06 \$A2.00+0.00 
follows from the $L^{p}$ boundedness of the maximally modulated Hilbert transform

$$
H_{*}(f)=\sup _{\xi \in \mathbb{R}}\left|H\left(M^{\xi} f\right)\right|,
$$

on $L^{p}(\mathbb{R})$, where $M^{\xi} f(x)=e^{2 \pi i \xi x} f(x)$ and $H$ is the Hilbert transform; the passage from the circle to the line here follows from the maximal transference theorem of Kenig and Tomas [11].

It is natural to investigate analogous reductions of the problem of convergence of multilinear Fourier series on $\mathbb{T}^{n} \times \cdots \times \mathbb{T}^{n}$ to the boundedness of multilinear operators on $\mathbb{R}^{n} \times \cdots \times \mathbb{R}^{n}$. It turns out that such reductions are possible and are easy consequences of a rich theory of multilinear transference. Multilinear transference has been studied by (in chronological order) Murray [15], Grafakos and Weiss [9], Fan and Sato [6], Blasco [1], and Blasco and Villaroya [2]. These articles are concerned with transference of operators that are linear in each variable. In this work we discuss transference of maximal multilinear operators analogous to that obtained by Kenig and Tomas [11] for maximal linear operators.

As an application of transference (and some basic functional analysis), one can use the boundedness of the bilinear Hilbert transforms

$$
H_{\alpha}\left(f_{1}, f_{2}\right)(x)=\frac{1}{\pi} \mathrm{p} \cdot \mathrm{v} \cdot \int_{\mathbb{R}} f_{1}(x-t) f_{2}(x+\alpha t) \frac{\mathrm{d} t}{t},
$$

obtained by Lacey and Thiele $[12,13]$ to deduce the $L^{p}$ convergence of the bilinear Fourier series

$$
\sum_{\substack{|m+n|<N \\|m-\alpha n|<N}} \hat{g}_{1}(m) \hat{g}_{2}(n) e^{2 \pi i(m+n) x}
$$

as $N \rightarrow \infty$ and vice versa. Here $\alpha$ is a fixed real number, $g_{1}, g_{2}$ are functions on the circle $\mathbb{T}$, and $f_{1}, f_{2}$ functions on the line. We note that the aforementioned convergence can also be obtained via the boundedness of the bilinear conjugate function obtained in Fan and Sato [6] using transference. Likewise, we can use maximal multilinear transference to obtain almost everywhere convergence for multilinear Fourier series. Details on these applications will be given at the end of this paper.

We will be working with indices $1 \leq p_{1}, \ldots, p_{m} \leq \infty$ such that

$$
\frac{1}{p}=\frac{1}{p_{1}}+\frac{1}{p_{2}}+\cdots+\frac{1}{p_{m}}>0 .
$$

We say that a function $B \in L^{\infty}\left(\left(\mathbb{R}^{n}\right)^{m}\right)$ is an $m$-linear multiplier, or lies in $\mathscr{M}_{p_{1}, p_{2} \ldots ., p_{m}, p}\left(\mathbb{R}^{n}\right)$, if the $m$-linear operator

$$
\begin{aligned}
& T_{B}\left(f_{1}, \ldots, f_{m}\right)(x) \\
& \quad=\int_{\left(\mathbb{R}^{n}\right)^{m}} B\left(\xi_{1}, \ldots, \xi_{m}\right) \hat{f}_{1}\left(\xi_{1}\right) \cdots \hat{f}_{m}\left(\xi_{m}\right) e^{2 \pi i\left(\sum, \xi_{j}\right) x} \mathrm{~d} \xi_{1} \ldots \mathrm{d} \xi_{m}
\end{aligned}
$$


satisfies, for some constant $C$, the estimate

$$
\left\|T_{B}\left(f_{1}, \ldots, f_{m}\right)\right\|_{L^{p}} \leq C\left\|f_{1}\right\|_{L^{p_{1}}} \ldots\left\|f_{m}\right\|_{L^{p m}}
$$

for all smooth compactly supported functions $f_{j}$ on $\mathbb{R}^{n}$. When all $p_{j}<\infty$ this means that $T_{B}$ admits a bounded extension from $L^{p_{1}}\left(\mathbb{R}^{n}\right) \times \cdots \times L^{p_{m}}\left(\mathbb{R}^{n}\right)$ to $L^{P}\left(\mathbb{R}^{n}\right)$.

We define the class $\mathscr{M}_{p_{1}, p_{2} \ldots ., p_{m}, p}\left(\mathbb{T}^{n}\right)$ in the same way. (We identify $\mathbb{T}$ with $[0,1]$.) We say that a sequence $b \in L^{\infty}\left(\left(\mathbb{Z}^{n}\right)^{m}\right)$ belongs to $\mathscr{M}_{p_{1}, p_{2}, \ldots . p_{m}, p}\left(\mathbb{T}^{n}\right)$, or is an $m$-linear multiplier on $\mathbb{T}^{n}$, if the operator

$$
S_{b}\left(g_{1}, \ldots, g_{m}\right)(x)=\sum_{k \in\left(\mathbb{Z}^{n}\right)^{m}} b\left(k_{1}, \ldots, k_{m}\right) \hat{g}_{1}\left(k_{1}\right) \cdots \hat{g}_{m}\left(k_{m}\right) e^{2 \pi i\left(\sum_{j} k_{j}\right) x},
$$

initially defined for trigonometric polynomials $g_{j}$, extends to a bounded operator from $L^{p_{1}}\left(\mathbb{T}^{n}\right) \times \cdots \times L^{p_{m}}\left(\mathbb{T}^{n}\right)$ to $L^{p}\left(\mathbb{T}^{n}\right)$. The spaces $\mathscr{M}_{p_{1}, p_{2}, \ldots, p_{m}, p}\left(\mathbb{R}^{n}\right)$ and $\mathscr{M}_{p_{1}, p_{2}, \ldots, p_{m}, p}\left(\mathbb{T}^{n}\right)$ are easily seen to be Banach spaces (or quasi-Banach spaces when $p<1$ ) with respect to the norms (respectively quasi-norms when $p<1$ ) defined by the corresponding operator norms. We will use the notation

$$
\begin{aligned}
& \|B\|_{\mathscr{M}_{p_{1}, p_{2} \ldots \ldots, p_{m}, p^{(}}\left(\mathbb{R}^{n}\right)}=\left\|T_{B}\right\|_{L^{p_{1}}\left(\mathbb{R}^{n}\right) \times \cdots \times L^{p_{m}}\left(\mathbb{R}^{n}\right) \rightarrow L^{p}\left(\mathbb{R}^{n}\right)},
\end{aligned}
$$

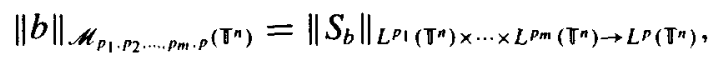

for these multiplier and operator norms.

We introduce the dilation operator $D^{R} f(x)=f(R x)$ for $R>0$ whenever $f$ is a function on $\mathbb{R}^{n}$. The following proposition summarizes a few basic properties of multilinear multipliers. The simple proof is omitted.

Proposition 1.1. Let $b_{1}, b_{2} \in \mathscr{M}_{p_{1}, p_{2}, \ldots . p_{m}, p}\left(\mathbb{R}^{n}\right)$ and $b \in \mathscr{M}_{p_{i}}\left(\mathbb{R}^{n}\right)$ for some $1 \leq$ $p_{1}, \ldots, p_{n} \leq \infty$ and $0<p<\infty$ satisfying $1 / p_{1}+\cdots+1 / p_{m}=1 / p$. Then

(a) $T_{b_{1}}+T_{b_{2}}=T_{b_{1}+b_{2}} \in \mathscr{M}_{p_{1}, p_{2} \ldots, p_{m}, p}$.

(b) $T_{b_{1}}\left(\cdot, \ldots, T_{b}(\cdot), \ldots, \cdot\right)=T_{b_{1} \otimes_{i} b} \in \mathscr{M}_{p_{1}, p_{2}, \ldots, p_{m}, p}$, where $\otimes_{i}$ represents product in the variable $i$, that is, $\left(b_{1} \otimes_{i} b\right)\left(x_{1}, \ldots, x_{m}\right)=b_{1}\left(x_{1}, \ldots, x_{m}\right) b\left(x_{i}\right)$.

(c) The dilation operator $D^{R}$ leaves the norm of a multiplier invariant, that is,

$$
\left\|D^{R} b_{1}\right\|_{\mathscr{M}_{p_{1}, p_{2} \ldots, p_{m}, \rho}}=\left\|b_{1}\right\|_{\mathscr{M}_{p_{1}, p_{2} \ldots, p_{m}, p}} .
$$

\section{Transference of maximal multipliers}

The main result of this section, Theorem 2.2, concerns transference of maximal multipliers. This theorem will be a key element in obtaining almost everywhere convergence for certain multilinear Fourier series. A similar result is mentioned as a 
remark (without proof) in Fan and Sato [6] for maximal dilations of multipliers. For the applications we have in mind, we need a slightly stronger version of this theorem that allows an arbitrary family of multipliers.

We fix a set $\Gamma$ and a set of $b_{\alpha} \in L^{\infty}\left(\left(\mathbb{R}^{n}\right)^{m}\right)$ indexed by $\alpha \in \Gamma$. We also fix indices $p_{1}, \ldots, p_{m}$ that atisfy (2) and we assume that $T_{b_{\alpha}}$ is a bounded $m$-linear operator from $L^{p_{1}}\left(\mathbb{R}^{n}\right) \times \cdots \times L^{p_{m}}\left(\mathbb{R}^{n}\right)$ to $L^{p}\left(\mathbb{R}^{n}\right)$. Then, for $f_{j} \in L^{p_{j}}\left(\mathbb{R}^{n}\right)$, we set

$$
N\left(f_{1}, \ldots, f_{m}\right)(x)=\sup _{\alpha \in \Gamma}\left|T_{b_{\alpha}}\left(f_{1}, \ldots, f_{m}\right)(x)\right| .
$$

We make a similar assumption for $S_{b_{\alpha}}$ and for $g_{i} \in L^{p_{i}}\left(\mathbb{T}^{n}\right)$, we set

$$
M\left(g_{1}, \ldots, g_{m}\right)(x)=\sup _{\alpha \in \Gamma}\left|S_{b_{\alpha}}\left(g_{1}, \ldots, g_{m}\right)(x)\right| .
$$

We will need the following lemma from the measure theory whose easy proof is omitted (see [7]).

LEMMA 2.1. Let A be a family of measurable functions on a measure space $X$. Suppose that

$$
\sup _{(F \subset A: F \text { finite) }}\left\|\sup _{f \in F} f\right\|_{L^{\rho}(X)} \leq C<\infty .
$$

Then for every $f \in A$ there is a measurable function $g_{f}$, such that $f=g_{f}$ a.e. and

$$
\left\|\sup _{f \in A} g_{f}\right\|_{L^{p}(X)} \leq C .
$$

We introduce the following notation $G_{p, \varepsilon}(x)=e^{-\pi|\varepsilon x|^{2} / p}$ which will be used repeatedly in the sequel. We note that for all continuous functions $g$ on $\mathbb{T}^{n}$ we have

$$
\lim _{\varepsilon \rightarrow 0} \varepsilon^{n} \int_{\mathbb{R}^{n}} g(x) G_{1, \varepsilon}(x) \mathrm{d} x=\int_{\mathbb{T}^{n}} g(x) \mathrm{d} x .
$$

We now state and prove the main result of this section, a transference theorem for maximal multilinear multipliers. In the case $m=1$, a slightly weaker version of this theorem was obtained by Kenig and Tomas [11].

THEOREM 2.2. Let $1 \leq p_{1}, \ldots, p_{n}<\infty, 0<p<\infty$, where $\sum_{i=1}^{m} 1 / p_{i}=1 / p$, and let $b_{\alpha} \in L^{\infty}\left(\left(\mathbb{R}^{n}\right)^{m}\right)$, where $\alpha \in \Gamma$. Assume that every $b_{\alpha}$ has a Lebesgue point at every $k \in\left(\mathbb{Z}^{n}\right)^{m}$. Suppose that for all $f_{j} \in L^{p_{j}}\left(\mathbb{R}^{n}\right)$ we have

$$
\left\|N\left(f_{1}, \ldots, f_{m}\right)\right\|_{L^{r}\left(\mathbb{R}^{n}\right)} \leq C\left\|f_{1}\right\|_{L^{r_{1}}\left(\mathbb{R}^{n}\right)} \cdots\left\|f_{m}\right\|_{L^{r_{m}\left(\mathbb{R}^{n}\right)}} .
$$

Then for all $g_{j} \in L^{p_{j}}\left(\mathbb{T}^{n}\right)$ we have

$$
\left\|M\left(g_{1}, \ldots, g_{m}\right)\right\|_{L^{p}\left(\mathbb{J}^{n}\right)} \leq C\left\|g_{1}\right\|_{L^{p_{1}}\left(\mathbb{I}^{n}\right)} \cdots\left\|g_{m}\right\|_{L^{p_{m}\left(\mathbf{I}^{n}\right)}} .
$$


Proof. Let us fix $\alpha_{1}, \ldots, \alpha_{k}$ in the index set $\Gamma$. In view of Lemma 2.1, it will be enough to prove the boundedness of the following operator

$$
M^{0}\left(Q_{1}, \ldots, Q_{m}\right)(x)=\sup _{b_{\alpha_{1}} \ldots, b_{\alpha_{k}}}\left|S_{b_{\alpha_{i}}}\left(Q_{1}, \ldots, Q_{m}\right)(x)\right| .
$$

In analogy, for trigonometric polynomials $Q_{1}, \ldots Q_{m}$, we define

$$
N^{0}\left(Q_{1}, \ldots, Q_{m}\right)(x)=\sup _{b_{\alpha_{1}}, \ldots b_{\alpha_{k}}}\left|T_{b_{\alpha_{i}}}\left(f_{1}, \ldots, f_{m}\right)(x)\right| .
$$

We shall first obtain the boundedness of $M^{0}$ for trigonometric polynomials $Q_{j}$. We observe that for linear monomials $P_{j}(x)=e^{2 \pi i k_{j} x}$ we have

$$
\begin{aligned}
& S_{b}\left(P_{1}, \ldots, P_{m}\right)(x) G_{p, \varepsilon}(x) \\
& \quad=C_{0} \varepsilon^{-m n} \int_{\left(\mathbb{R}^{n}\right)^{m}} b\left(k_{1}, \ldots, k_{m}\right) \prod_{j=1}^{m} \exp \left(-\frac{\pi p_{j}}{\varepsilon^{2}}\left|\xi_{j}-k_{j}\right|^{2}\right) e^{2 \pi i\left(\sum_{j} \xi_{j}\right) x} \mathrm{~d} \xi,
\end{aligned}
$$

where $C_{0}=\sqrt{p_{1}^{n} \cdots p_{m}^{n}}$. If we set $g_{j}(x)=P_{j}(x) G_{p_{j}, \varepsilon}(x)$, we can write

$$
\begin{aligned}
& T_{b}\left(g_{1}, \ldots, g_{m}\right)(x) \\
& \quad=C_{0} \varepsilon^{-m n} \int_{\left(\mathbb{R}^{n}\right)^{m}} b\left(\xi_{1}, \ldots, \xi_{m}\right) \prod_{j=1}^{m} \exp \left(-\frac{\pi p_{j}}{\varepsilon^{2}}\left|\xi_{j}-k_{j}\right|^{2}\right) e^{2 \pi i\left(\sum_{j} \xi_{j}\right) x} \mathrm{~d} \xi
\end{aligned}
$$

and compare the two operators as follows:

$$
\begin{aligned}
& \left|S_{b}\left(P_{1}, \ldots, P_{m}\right)(x) G_{p, \varepsilon}(x)-P_{b}\left(g_{1}, \ldots, g_{m}\right)(x)\right| \\
& \quad \leq C^{\prime}\|b\|_{\infty} \int_{\left\{\xi \in\left(\mathbb{R}^{n}\right)^{m}:|\xi| \geq r\right\}} e^{-\pi|\xi|^{2}} \mathrm{~d} \xi \\
& \quad+C^{\prime} \varepsilon^{-m n} \int_{\left\{\eta \in\left(\mathbb{R}^{n}\right)^{m}:|\eta|<r \varepsilon\right\}}\left|b\left(k_{1}, \ldots, k_{m}\right)-b\left(\frac{\eta_{1}}{\sqrt{p_{1}}}+k_{1}, \ldots, \frac{\eta_{m}}{\sqrt{p_{m}}}+k_{m}\right)\right| \mathrm{d} \eta,
\end{aligned}
$$

where $r>0$ is arbitrary. The first term above tends to 0 as $r \rightarrow \infty$ while the second one tends to 0 as $\epsilon \rightarrow 0$ whenever $k$ is a Lebesgue point of $b$. We can extend the same estimate to trigonometric polynomials $Q_{j}$ by linearity. Taking the supremum, we obtain

$$
\left|M^{0}\left(Q_{1}, \ldots, Q_{m}\right)(x) G_{p, \varepsilon}-N^{0}\left(Q_{1} G_{p_{1}, \varepsilon}, \ldots, Q_{m} G_{p_{m}, \varepsilon}\right)(x)\right| \leq o_{1 / r}(1)+r^{m n} o_{\varepsilon}(1) .
$$

Using (5) we deduce

$$
\begin{aligned}
& \int_{\mathbb{J}^{n}} \sup _{b_{\alpha_{1}} \ldots . b_{a_{k}}}\left|S_{b_{\alpha_{i}}}\left(Q_{1}, \ldots, Q_{m}\right)(x)\right|^{p} \mathrm{~d} x \\
& \quad=\left.\lim _{\varepsilon \rightarrow 0} \varepsilon^{n} \int_{\mathbb{R}^{n} b_{\alpha_{1}}, \ldots, b_{\alpha_{k}}} \sup _{b_{\alpha_{\alpha_{i}}}}\left(Q_{1}, \ldots, Q_{m}\right)(x) G_{p . \varepsilon}(x)\right|^{p} \mathrm{~d} x .
\end{aligned}
$$


The function $M^{0}\left(Q_{1}, \ldots, Q_{m}\right)$ is bounded by some constant $K$, so

$$
\begin{aligned}
& \lim _{\varepsilon \rightarrow 0} \varepsilon^{n} \int_{\mathbb{R}^{n}} \sup _{b_{\alpha_{1}} \ldots, b_{\alpha_{k_{k}}}}\left|S_{b_{\alpha_{i}}}\left(Q_{1}, \ldots, Q_{m}\right)(x) G_{p, \varepsilon}(x)\right|^{p} \mathrm{~d} x \\
& \quad \leq K o_{1 / R}(1)+\lim _{\varepsilon \rightarrow 0} \varepsilon^{n} \int_{\mathbb{R}^{n} \cap|| x|\leq R / \varepsilon|} \sup _{b_{\alpha_{1}}, \ldots, b_{\alpha_{k}}}\left|S_{b_{\alpha_{i}}}\left(Q_{1}, \ldots, Q_{m}\right)(x) G_{p, \varepsilon}(x)\right|^{p} \mathrm{~d} x .
\end{aligned}
$$

After we deduce the estimate

$$
\begin{aligned}
& \varepsilon^{n} \int_{|x| \leq R / \varepsilon} \sup _{b_{\alpha_{1}}, \ldots, b_{a_{k}}}\left|S_{b_{\alpha_{i}}}\left(Q_{1}, \ldots, Q_{m}\right)(x) G_{p, \varepsilon}(x)\right|^{p} \mathrm{~d} x \\
& \leq R^{n}\left(o_{1 / r}(1)+r^{m n} o_{\varepsilon}(1)\right) \\
&\left.\quad+\varepsilon^{n} \int_{\mathbb{R}^{n} b_{a_{1}} \ldots \ldots b_{\alpha_{k}}} \sup _{b_{b_{i}}} \mid Q_{1} G_{p_{1}, \varepsilon}, \ldots, Q_{m} G_{p_{m}, \varepsilon}\right)\left.(x)\right|^{p} \mathrm{~d} x
\end{aligned}
$$

we take $R$ and $r$ such that the first term above is negligible and finally obtain

$$
\begin{aligned}
& \left\|M^{0}\left(Q_{1}, \ldots, Q_{m}\right)\right\|_{L^{p}\left(\mathbb{U}^{n}\right)} \leq \limsup _{\varepsilon \rightarrow 0} \varepsilon^{n}\left\|N\left(Q_{1} G_{p_{1}, \varepsilon}, \ldots, Q_{m} G_{p_{m}, \varepsilon}\right)\right\|_{L^{r}\left(\mathbb{T}^{n}\right)} \\
& \leq C \limsup _{\varepsilon \rightarrow 0} \varepsilon^{n / p_{1}}\left\|Q_{1} G_{p_{1}, \varepsilon}\right\|_{L^{p_{1}}} \cdots \varepsilon^{n / p_{m}}\left\|Q_{m} G_{p_{m}, \varepsilon}\right\|_{L^{p_{m}}} \varepsilon^{n / p^{\prime}} \\
& \leq C\left\|Q_{1}\right\|_{L^{p 1}} \cdots\left\|Q_{m}\right\|_{L^{p m}} .
\end{aligned}
$$

This proves that the operator $M^{0}$ is bounded for trigonometric polynomials.

To extend the boundedness to general $L^{p_{j}}$ functions $g_{j}$, we first recall that the linear operator $S_{b_{\alpha}}$ is well defined and bounded on $L^{p_{1}} \times \cdots \times L^{p_{m}}$ for any $\alpha \in \Gamma$. This implies that whenever trigonometric polynomials $Q_{j, l} \rightarrow g_{j}$ in $L^{p_{j}}\left(\mathbb{J}^{n}\right)$, we have

$$
S_{b_{\alpha}}\left(Q_{1 . l}, \ldots, Q_{m . l}\right) \rightarrow S_{b_{\alpha}}\left(g_{1}, \ldots, g_{m}\right)
$$

in $L^{p}$. We can now can use the trivial estimate

$$
\begin{aligned}
& \left|M^{0}\left(g_{1}, \ldots, g_{m}\right)(x)-M^{0}\left(Q_{1, l}, \ldots, Q_{m, l}\right)(x)\right| \\
& \quad \leq \sum_{i=1}^{k}\left|S_{b_{\alpha_{i}}}\left(Q_{1, l}, \ldots, Q_{m, l}\right)(x)-S_{b_{\alpha_{i}}}\left(g_{1}, \ldots, g_{m}\right)(x)\right|
\end{aligned}
$$

and take the $L^{p}$ norm to obtain the required estimate for general functions $g_{j} \in$ $L^{n}$.

We make a couple of remarks. It is possible to define the multilinear multiplier even in the case when some $p_{i}=\infty$. The multiplier then, of course, extends only to the closure of the set $C_{0}^{\infty}$ in $L^{\infty}$. It is possible to prove the above transference result in 
this setting, using an arbitrary cutoff function in place of $G_{\varepsilon, p_{i}}$. When $p \geq 1$, the proof can be trivially extended to regulated maximal multipliers, where $b_{\alpha}$ is regulated, if $b_{\alpha}(x)=\lim _{\phi_{n}} \phi_{n} * b_{\alpha}$ for any point $x \in \mathbb{Z}^{n m}$ for some approximate identity $\phi_{n}$. Key to this is the following lemma, which has been proved for bilinear multipliers in [1].

LEMMA 2.3. Assume the hypotheses of Theorem 2.2 and also that $p \geq 1$. Let $\Phi$ be in $L^{1}\left(\mathbb{R}^{m n}\right)$. Then for any $b_{1}=b_{\alpha_{1}} * \Phi, \ldots, b_{k}=b_{\alpha_{k}} * \Phi$ we have

$$
\left\|\sup _{b_{1}, \ldots, b_{k}}\left|T_{b_{i}}\left(q_{1}, \ldots, q_{m}\right)(x)\right|\right\|_{L^{p}\left(\mathbb{R}^{n}\right)} \leq C\|\Phi\|_{L^{1}\left(\mathbb{R}^{n}\right)}
$$

for Schwartz functions $q_{1}, \ldots, q_{m}$, where $C$ is the constant in Theorem 2.2.

ProOF. To prove this result, we need to use the linearization introduced in [11]. Clearly, if we write

$$
\begin{aligned}
\left\|\sup _{b_{1} \ldots, b_{k}}\left|T_{b_{i}}\left(q_{1}, \ldots, q_{m}\right)(x)\right|\right\|_{L^{p}\left(\mathbb{R}^{n}\right)} \\
\quad=\|\|\left(T_{b_{1}}\left(q_{1}, \ldots, q_{m}\right), \ldots, T_{b_{k}}\left(q_{1}, \ldots, q_{m}\right)\right)\left\|_{r_{k}^{\infty}}\right\|_{L^{p}\left(\mathbb{R}^{n}\right)},
\end{aligned}
$$

we can express the second norm as the supremum over all Schwartz functions $h_{1}, \ldots, h_{k}$ with $\left\|\sum_{i}\left|h_{i}\right|\right\|_{L^{p^{\prime}}\left(\mathbb{R}^{n}\right)} \leq 1$ of the expression

$$
\left|\int_{\mathbb{R}^{n}} \sum_{i} T_{b_{i}}\left(q_{1}, \ldots, q_{m}\right)(x) \overline{h_{i}(x)} \mathrm{d} x\right| .
$$

By Parseval's identity, this is equal to

$$
\int_{\mathbb{R}^{n m m}} \sum_{i} b_{i}\left(\xi_{1}, \ldots, \xi_{m}\right) \hat{q}_{1}\left(\xi_{1}\right) \cdots \hat{q}_{k}\left(\xi_{m}\right) \overline{\hat{h}_{i}\left(\sum_{j} \xi_{j}\right)} \mathrm{d} \xi_{1} \cdots \mathrm{d} \xi_{m} .
$$

The claim then follows by expressing $b_{i}$ as $b_{\alpha_{i}} * \Phi$, and applying the assumption that the maximal operator $N$ is bounded on products of Schwartz functions.

To obtain maximal transference in the opposite direction, one has to impose some additional condition on the set of the multipliers. For example, a standard condition is that the set of multipliers $\left\{b_{\alpha}\right\}_{\alpha}$ contains all dilations of its elements.

THEOREM 2.4. Let $1 \leq p_{1}, \ldots, p_{m}<\infty, 0<p<\infty$, where $\sum_{j=1}^{m} 1 / p_{j}=1 / p$, and let $b_{\alpha} \in L^{\infty}\left(\left(\mathbb{R}^{n}\right)^{m}\right)$, where $\alpha \in \Gamma$. Assume for every $b_{\alpha}$ and $R>0$ we have a $\beta \in \Gamma$ such that $b_{\alpha}=D^{R} b_{\beta}$. Let any $b_{\alpha}$ has a Lebesgue point at every $k \in\left(\mathbb{Z}^{n}\right)^{m}$ and let us assume that it is Riemann integrable over any rectangle. 
Suppose that for all functions $g_{j} \in L^{p_{j}}\left(\mathbb{J}^{n}\right)$ we have

$$
\left\|M\left(g_{1}, \ldots, g_{m}\right)\right\|_{L^{p}\left(\mathbb{J}^{n}\right)} \leq C\left\|g_{1}\right\|_{L^{p_{1}}\left(\mathbf{T}^{n}\right)} \cdots\left\|g_{m}\right\|_{L^{\rho_{m}\left(\mathbf{T}^{n}\right)}} .
$$

Then for all $f_{j} \in L^{p_{j}}\left(\mathbb{R}^{n}\right)$ we have

$$
\left\|N\left(f_{1}, \ldots, f_{m}\right)\right\|_{L^{p}\left(\mathbb{R}^{n}\right)} \leq C\left\|f_{1}\right\|_{L^{p_{1}}\left(\mathbb{R}^{n}\right)} \cdots\left\|f_{m}\right\|_{L^{p_{m}\left(\mathbb{R}^{n}\right)}} .
$$

Proof. Again, in view of the Lemma 2.1, it is enough to prove the boundedness of the operators $N^{0}$. Moreover, a limiting argument similar to that in Theorem 2.2 shows that it is enough to work with smooth compactly supported functions $a_{k}$. One can check that for any Riemann integrable bounded function $b$ we have

$$
\lim _{R \rightarrow \infty} D^{R^{-1}} S_{D^{R-1} b}\left(D^{R} a_{1}, \ldots, D^{R} a_{m}\right)(x)=T_{b}\left(a_{1}, \ldots, a_{m}\right)(x),
$$

where the right-hand side (which is well defined for large $R$ ) is a Riemann sum. For the maximal operators, this implies

$$
N^{0}\left(a_{1}, \ldots, a_{m}\right)(x) \leq \liminf _{R \rightarrow \infty} D^{R^{-1}} M\left(D^{R} a_{1}, \ldots, D^{R} a_{m}\right)(x)
$$

and the claim follows.

\section{Tools to study convergence}

Before we turn to applications of transference of maximal multipliers to convergence of $m$-linear Fourier series, we discuss a couple of useful results in the study of convergence. We begin with a general theorem that formulates an equivalent condition for the $L^{p}$ convergence of multilinear multiplier operators on the torus.

THEOREM 3.1. Fix $0<p<\infty$ and $\sum_{j=1}^{m} 1 / p_{j}=1 / p, 1 \leq p_{j}<\infty$. Suppose that for each $R>0$ there is a compactly supported sequence $b_{R} \in l^{\infty}\left(\left(\mathbb{Z}^{n}\right)^{m}\right)$ and a sequence $b \in l^{\infty}\left(\left(\mathbb{Z}^{n}\right)^{m}\right)$ such that for any $k \in\left(\mathbb{Z}^{n}\right)^{m}$ we have $b_{R}(k) \stackrel{R \rightarrow \infty}{\longrightarrow} b(k)$. Then the sequence $S_{b_{R}}\left(g_{1}, \ldots, g_{m}\right)$ converges in $L^{p}\left(\mathbb{T}^{n}\right)$ for any $g_{j} \in L^{p_{j}}\left(\mathbb{T}^{n}\right)$ if and only if there exists a constant $K<\infty$ such that

$$
\sup _{R>0}\left\|b_{R}\right\|_{\mathscr{H}_{p_{1}, p_{2} \ldots . ., p_{m}, p}} \leq K .
$$

Moreover, if (6) holds for some $K<\infty$, we must have $\|b\|_{\mathscr{M}_{p_{1}, p_{2} \ldots \ldots p_{m} . \rho}} \leq K$ and $S_{b_{R}}\left(g_{1}, \ldots, g_{m}\right) \rightarrow S_{b}\left(g_{1}, \ldots, g_{m}\right)$ in $L^{p}\left(\mathbb{T}^{n}\right)$ for all $g_{j} \in L^{p_{1}}\left(\mathbb{T}^{n}\right)$. (Here $S_{b}$ denotes the unique bounded extension of the same operator.) 
ProOF. We deduce (6) by a repeated application of the uniform boundedness theorem. Each operator $S_{b_{R}}$ corresponds to a compactly supported multiplier and is therefore bounded. From the convergence we see that for each $\left\{g_{j}\right\}_{j}$ we have a constant $C_{\left\{g_{j}\right\}_{j}}$ such that $\left\|S_{b_{R}}\left(g_{1}, \ldots, g_{m}\right)\right\|_{L^{p}} \leq C_{\left\{g_{j}\right\} ;}$ for any choice of $R$. Now we fix $g_{2}, \ldots, g_{m}$ and apply the uniform boundedness theorem on the family of linear operators $S_{b_{R}}\left(\cdot, g_{2}, \ldots, g_{m}\right)$, indexed by $R$. This gives us a constant $C_{g_{2}, \ldots, g_{m}}$,

$$
\left\|S_{b_{R}}\left(\cdot, g_{2}, \ldots, g_{m}\right)\right\|_{L^{\rho_{1} \rightarrow L^{r}}} \leq C_{g_{2}, \ldots, g_{m}} .
$$

In other words, we obtained a family of operators $S_{b_{R}}\left(g_{1} /\left\|g_{1}\right\|_{L^{p_{1}}}, \cdot, \ldots, g_{m}\right)$ indexed by $R$ and $g_{1}$. We use the uniform boundedness in the second variable and proceed by induction. This way we deduce the existence of a constant $K<\infty$ such that

$$
\left\|S_{b_{R}}\left(g_{1} /\left\|g_{1}\right\|_{L^{p_{1}}}, \ldots, g_{m} /\left\|g_{m}\right\|_{L^{p_{m}}}\right)\right\|_{L^{p}} \leq K .
$$

Let us now assume (6). Then clearly for any choice of trigonometric polynomials $\left\{Q_{j}\right\}_{j}$, Fatou's lemma gives

$$
\left\|S_{b}\left(Q_{1}, \ldots, Q_{m}\right)\right\|_{L^{p}} \leq \liminf _{R \rightarrow \infty}\left\|S_{b_{R}}\left(Q_{1}, \ldots, Q_{m}\right)\right\|_{L^{p}} \leq K\left\|Q_{1}\right\|_{L^{p_{1}}} \cdots\left\|Q_{m}\right\|_{L^{p^{m}}},
$$

which means that $S_{b}$ extends to a bounded operator on $L^{p_{1}} \times \cdots \times L^{p_{m}}$ with norm bounded by $K$.

Fix now $g_{j} \in L^{p_{j}}\left(\mathbb{T}^{n}\right)$ for each $1 \leq j \leq m$. For any $\varepsilon>0$, we may take trigonometric polynomials $\left\{Q_{j}\right\}$ such that $\left\|Q_{j}-g_{j}\right\|_{L^{p}} \leq \varepsilon$. The Fourier transform of a trigonometric polynomial is compactly supported, which means

$$
\left\|S_{b}\left(Q_{1}, \ldots, Q_{m}\right)-S_{b_{R}}\left(Q_{1}, \ldots, Q_{m}\right)\right\|_{L^{p}} \rightarrow 0 .
$$

We can pick $R_{0}$ such that the above quantity is less than $\varepsilon$ for $R>R_{0}$ and then write

$$
\begin{aligned}
&\left\|S_{b}\left(g_{1}, \ldots, g_{m}\right)-S_{b_{R}}\left(g_{1}, \ldots, g_{m}\right)\right\|_{L^{p}} \\
& \leq C_{p}\left(\left\|S_{b_{R}}\left(g_{1}, \ldots, g_{m}\right)-S_{b_{R}}\left(Q_{1}, \ldots, Q_{m}\right)\right\|_{L^{p}}\right. \\
&+\left\|S_{b_{R}}\left(Q_{1}, \ldots, Q_{m}\right)-S_{b}\left(Q_{1}, \ldots, Q_{m}\right)\right\|_{L^{p}} \\
&\left.+\left\|S_{b}\left(Q_{1}, \ldots, Q_{m}\right)-S_{b}\left(g_{1}, \ldots, g_{m}\right)\right\|_{L^{p}}\right) .
\end{aligned}
$$

The middle term on the right is controlled by $\varepsilon$, while the remaining two can be estimated using multilinearity by the usual transformation

$$
\begin{aligned}
S_{b}\left(Q_{1}, \ldots, Q_{m}\right)-S_{b}\left(g_{1}, \ldots, g_{m}\right) & \\
= & S_{b}\left(Q_{1}, \ldots, Q_{m}-g_{m}\right)+S_{b}\left(Q_{1}, \ldots, Q_{m-1}-g_{m-1}, g_{m}\right) \\
& +\cdots-S_{b}\left(g_{1}-Q_{1}, \ldots, g_{m}\right) .
\end{aligned}
$$

These terms all have $L^{p}$ norm estimated by some constant multiple of $K \varepsilon$. The same works for $S_{b_{R}}$. The result follows by triangle inequality (or quasi-triangle inequality when $p<1$ ). 
Next we obtain a result allowing us to derive almost everywhere convergence for multilinear operators from the boundedness of a corresponding maximal operator. Let $\left(X_{j}, \mu_{j}\right), 1 \leq j \leq m,(Y, v)$ be measure spaces and let $0<p_{j} \leq \infty, 0<q<\infty$. Suppose that $\mathscr{D}$ is a dense subspace of $L^{p_{j}}(X, \mu)$ for all $j$. Suppose that for every $\varepsilon>0, T_{\varepsilon}$ is an $m$-linear operator defined on $L^{p_{1}}\left(X_{1}, \mu_{1}\right) \times \cdots \times L^{p_{m}}\left(X_{m}, \mu_{m}\right)$ with values in the set of measurable functions on $Y$. Define a sublinear operator

$$
T_{*}\left(f_{1}, \ldots, f_{m}\right)=\sup _{\varepsilon>0}\left|T_{\varepsilon}\left(f_{1}, \ldots, f_{m}\right)\right| .
$$

Then we have the following result.

THEOREM 3.2. Suppose that for some $B>0$ and all $f_{j} \in L^{p_{j}}\left(X_{j}, \mu_{j}\right)$ we have

$$
\left\|T_{*}\left(f_{1}, \ldots, f_{m}\right)\right\|_{L^{q . x}} \leq B\left\|f_{1}\right\|_{L^{p_{1}}} \ldots\left\|f_{m}\right\|_{L^{p_{m}}}
$$

and that for all $h_{j} \in \mathcal{O}$

$$
\lim _{\xi \rightarrow 0} T_{F}\left(h_{1}, \ldots, h_{m}\right)=T\left(h_{1}, \ldots, h_{m}\right)
$$

exists and is finite v-a.e. and defines a multilinear operator on $\mathscr{D}^{m}$. Then for all $f_{j} \in L^{p}\left(X, \mu_{j}\right)$ the limit (8) exists and is finite $v$-a.e. and defines a bounded multilinear operator $T$ from $L^{p_{1}}\left(X_{1}\right) \times \cdots \times L^{p_{m}}\left(X_{m}\right)$ to $L^{q, \infty}(Y)$ that uniquely extends $T$ defined on $\mathscr{D}^{m}$.

ProOF. Given a tuple $\left(f_{1}, \ldots, f_{m}\right)$ in $L^{p_{1}} \times \cdots \times L^{p_{m}}$, we define its oscillation at the point $y \in Y$ as

$$
O\left(f_{1}, \ldots, f_{m}\right)(y)=\limsup _{\varepsilon \rightarrow 0} \limsup _{\theta \rightarrow 0}\left|T_{\varepsilon}\left(f_{1}, \ldots, f_{m}\right)(y)-T_{\theta}\left(f_{1}, \ldots, f_{m}\right)(y)\right| .
$$

We will show that for all $\left(f_{1}, \ldots, f_{m}\right)$ in $L^{p_{1}} \times \cdots \times L^{p_{m}}$ and $\delta>0$, we have

$$
v\left(\left\{y \in Y: O\left(f_{1}, \ldots, f_{m}\right)(y)>\delta\right\}\right)=0 .
$$

Once (9) is established, given $\left(f_{1}, \ldots, f_{m_{1}}\right)$ in $L^{p_{1}} \times \cdots \times L^{p_{m}}$, we obtain that $O\left(f_{1}, \ldots, f_{m}\right)(y)=0$ for $v$-almost all $y \in Y$, which implies that $T_{\varepsilon}\left(f_{1}, \ldots, f_{m}\right)(y)$ is Cauchy for $v$-almost all $y$ and it therefore converges $v$-a.e. to some multilinear operator $T\left(f_{1}, \ldots, f_{m}\right)(y)$ as $\varepsilon \rightarrow 0$ that extends $T$ defined on $\mathscr{Q}^{m}$.

To approximate $O\left(f_{1}, \ldots, f_{m}\right)$ we use density. Given $0<\eta<1$, find $g_{j} \in \mathscr{D}$ such that $\left\|f_{j}-g_{j}\right\|_{L^{n},}<\eta$. It is easy to see that for some constant $C$ we have

$$
O\left(f_{1}, \ldots, f_{m}\right) \leq O\left(g_{1}, \ldots, g_{m}\right)+C \sum O\left(\phi_{1}, \ldots, \phi_{m}\right) \quad v \text {-a.e., }
$$

where $\phi_{j}$ is either $f_{j}$ or $f_{j}-g_{j}$ and the sum is taken over all finitely many possible combinations of expressions of this sort in which at least one $\phi_{k}$ is $f_{k}-g_{k}$. Since 
$T_{\varepsilon}\left(g_{1}, \ldots, g_{m}\right) \rightarrow T\left(g_{1}, \ldots, g_{m}\right) v$-a.e., it follows that $O\left(g_{1}, \ldots, g_{m}\right)=0$ v-a.e. We can therefore pointwise control the oscillation $O\left(f_{1}, \ldots, f_{m}\right)$ by a sum of oscillations of tuples of functions in which at least one entry has small norm.

Now for any $\delta>0$ and any tuple $\left(\phi_{1}, \ldots, \phi_{m}\right)$ as above we have

$$
\begin{aligned}
v\left(\left\{O\left(\phi_{1}, \ldots, \phi_{m}\right)>\delta\right\}\right) & \leq v\left(\left\{O\left(\phi_{1}, \ldots, \phi_{m}\right)>\delta\right\}\right) \\
& \leq v\left(\left\{T_{*}\left(\phi_{1}, \ldots, \phi_{m}\right)>\delta / 2\right\}\right) \\
& \leq\left(2 B\left\|\phi_{1}\right\|_{L^{p_{1}}} \ldots\left\|\phi_{m}\right\|_{L^{p_{m}}} / \delta\right)^{q} \\
& \leq C\left(f_{1}, \ldots, f_{m}\right)(2 B / \delta)^{q} \eta^{q}
\end{aligned}
$$

where $C\left(f_{1}, \ldots, f_{m}\right)$ is a constant depending on the functions $f_{j}$. Letting $\eta \rightarrow 0$ and using (10), we deduce (9). We conclude that $T_{\varepsilon}\left(f_{1}, \ldots, f_{m}\right)$ is a Cauchy sequence and hence it converges $v$-a.e. to some $T\left(f_{1}, \ldots, f_{m}\right)$. Since $\left|T\left(f_{1}, \ldots, f_{m}\right)\right| \leq$ $\left|T_{*}\left(f_{1}, \ldots, f_{m}\right)\right|$, it follows that $T$ is a bounded operator (with norm at most $B$ ).

\section{Multilinear Fourier series}

We now discuss applications of the preceding results. We consider an open connected set $E \subset \mathbb{B}^{m n}$ which contains the point 0 in its interior. Define the $m$-linear Fourier partial sum

$$
P_{R}^{E}\left(g_{1}, \ldots, g_{m}\right)(x)=\sum_{\left(k_{1}, \ldots, k_{m}\right) \in R \cdot\left(E \cap \mathbb{Z}^{m+n}\right)} \hat{g}_{1}\left(k_{1}\right) \ldots \hat{g}_{m}\left(k_{m}\right) e^{2 \pi i\left(k_{1}+\cdots+k_{m}\right) \cdot x}
$$

which naturally converges to $g_{1}(x) \cdots g_{m}(x)$ whenever $g_{1}, \ldots, g_{m}$ are smooth functions on $\mathbb{J}^{n}$. The summation here is taken over all lattice points inside the $R$-fold dilate of the set $E$ and the convergence is understood as $R \rightarrow \infty$. We will use transference to study the $L^{p}$ and almost everywhere convergence of this series whenever the $g_{j}$ lie in some Lebesgue spaces. In view of Theorem 3.1, the $L^{p}$ convergence of $P_{R}^{E}$ is a consequence of the uniform boundedness of the family of multilinear operators $\left\{P_{R}^{E}\right\}_{R>0}$. Transferring these operators to $\mathbb{R}^{n}$, reduces matters to showing that $\chi_{E}$ lies in $\mathscr{M}_{p_{1} \ldots, p_{m}, p}\left(\mathbb{R}^{n}\right)$ (for the $L^{p}$ convergence problem) and that the maximal operator $\sup _{N>0}\left|T_{X_{N E} . E}\right|$ is bounded (for the almost everywhere convergence problem). Here $N \cdot E$ is an $N$-fold dilate of $E$ and $T_{B}$ is defined in (3).

We consider the case in which the set $E$ is a polygon in $\mathbb{R}^{2}$ with finitely many sides. We prove an easy geometric lemma, which allows us to write this polygon as a difference of finite unions of triangles.

LEMMA 4.1. Let $D \subset \mathbb{R}^{2}$ be a closed polygon with finitely many sides. Then we can find two finite sets $\mathscr{T}_{1}, \mathscr{T}_{2}$ of closed triangles each of which has two sides parallel to axes such that $\sum_{T \in \mathscr{T}_{1}} \chi_{T}-\sum_{T \in \mathscr{T}_{2}} \chi_{T}=\chi_{D}$ a.e. 
PROOF. Clearly, we can divide any polygon into finitely many triangles. Consider such a triangle and denote it $T$. If $T$ does not have any sides parallel to the first coordinate axis, the orthogonal projection of its vertices on the second axis consists of three distinct points and the straight line parallel to the first coordinate axis passing though the mi'dle of these three points splits $T$ into two triangles $T^{\prime}$ and $T^{\prime \prime}$ which have one side parallel to the first coordinate axis.

Let $T^{\prime}$ have vertices $A, B, C$, where the line $A B$ is parallel to the first coordinate axis. If no remaining side of $T^{\prime}$ is parallel to the second coordinate axis, let $X$ be the point of intersection of the line passing through $A$ and $B$ and of the line passing through the point $C$ and parallel to the second coordinate axis. The triangles $A X C$ and $B X C$ have two sides parallel to the coordinates, and we have

$$
\begin{array}{ll}
\chi_{A B C}=\chi_{A X C}+\chi_{B X C} \quad \text { or } \\
\chi_{A B C}=\chi_{A X C}-\chi_{B X C} \quad \text { or } \\
\chi_{A B C}=-\chi_{A X C}+\chi_{B X C} \text { a.e. }
\end{array}
$$

So this procedure splits $T$ to at most four triangles, which we place in $\mathscr{T}_{1}$ or $\mathscr{T}_{2}$ according to the sign they inherit by the previous identities.

We now discuss the problem of the convergence of bilinear Fourier series summed over lattice points inside dilates of polygons in $\mathbb{R}^{2}$. Let us fix such a polygon $D$. Apply Lemma 4.1 to obtain sets of triangles indexed by the sets $\mathscr{T}_{1}$ and $\mathscr{T}_{2}$. It follows from the work of Lacey and Thiele [12], [13] that the characteristic function of any triangle in $\mathbb{R}^{2}$ with no side parallel to the antidiagonal $y=-x$ lies in $\mathscr{M}_{p_{1}, p_{2}, p}(\mathbb{R})$ where $2 / 3<p<\infty, 1<p_{1}, p_{2} \leq \infty$ and $1 / p_{1}+1 / p_{2}=1 / p$. (If the triangle has a side parallel to the antidiagonal $y=-x$, then the same conclusion is valid with the additional restriction that $p>1$.)

Using Lemma 4.1 we conclude that the characteristic function of a polygon $D$ in $\mathbb{R}^{2}$ with no side parallel to the antidiagonal $y=-x$ is a bounded bilinear multiplier in $\mathscr{M}_{p_{1}, p_{2}, p}(\mathbb{R})$ where $2 / 3<p<\infty, 1<p_{1}, p_{2} \leq \infty$ and $1 / p_{1}+1 / p_{2}=1 / p$. Moreover, part (c) of Proposition 1.1 says that any dilate of $D$ is also a bounded bilinear multiplier (with the same norm). We can now take a suitable increasing sequence or $R_{n}$ such that $D$ dilated by $R_{n}$ contains no lattice point in its boundary and such that there is exactly one $R_{n}^{\prime}$ between $R_{n}$ and $R_{n+1}$ such that the dilate of $D$ by the amount $R_{n}^{\prime}$ has a lattice point in its boundary. We can also arrange so that the dilate of $D$ by $R_{1}$ contains only the zero lattice point. This choice of our sequence ensures that for any $R>0$ there is an $n$ such that $P_{R}^{D}=P_{R_{n}}^{D}$. Thus the characteristic function of any dilate of $D$ has a Lebesgue point at every lattice point and we can apply both the transference theorem in [6] and Theorem 3.1 to obtain the boundedness of each of the 
operators $P_{R_{n}}^{D}$. We conclude that $P_{R}^{D}\left(g_{1}, g_{2}\right) \rightarrow g_{1} g_{2}$ in $L^{p}(\mathbb{T})$ for any $g_{1} \in L^{p_{1}}(\mathbb{T})$ and $g_{2} \in L^{p_{2}}(\mathbb{T})$ where $2 / 3<p<\infty, 1<p_{1}, p_{2} \leq \infty$. Precisely we have the following.

THEOREM 4.2. Let $2 / 3<p<\infty, 1<p_{1}, p_{2}<\infty, 1 / p_{1}+1 / p_{2}=1 / p$ and $D \subset \mathbb{R}^{2}$ be a polygon that contains 0 in its interior and has no side parallel to the antidiagonal $y=-x$. Then for $g_{1} \in L^{p_{1}}(\mathbb{T})$ and $g_{2} \in L^{p_{2}}(\mathbb{T})$ we have

$$
P_{R}^{D}\left(g_{1}, g_{2}\right) \stackrel{R \rightarrow \infty}{\longrightarrow} g_{1} g_{2} \quad \text { in } L^{P}(\mathbb{T}) .
$$

Using a similar argument we can also obtain a theorem in which the summation is taken over lattice points in a dilate of a disc.

THEOREM 4.3. Let $1<p<2,2<p_{1}, p_{2}<\infty, 1 / p_{1}+1 / p_{2}=1 / p$ and let $U$ be the unit disc in $\mathbb{R}^{2}$. Then for $g_{1} \in L^{p_{1}}(\mathbb{T})$ and $g_{2} \in L^{p_{2}}(\mathbb{T})$ we have

$$
P_{R}^{U}\left(g_{1}, g_{2}\right) \stackrel{R \rightarrow \infty}{\longrightarrow} g_{1} g_{2} \text { in } L^{p}(\mathbb{T}) \text {. }
$$

Theorem 4.3 easily follows by applying the previous reasoning to the characteristic function of a disc in $\mathbb{R}^{2}$ and using the fact that this function is an $\mathscr{M}_{p_{1}, p_{2}, p}$ bilinear multiplier on $\mathbb{R} \times \mathbb{R}$. For the last result we refer to [8].

We now pass to an application of the maximal transference Theorem 2.2. Let $E$ be a polyhedron in $\mathbb{B}^{m}$ containing the origin. One would like to known whether the expressions $P_{R}^{E}\left(g_{1}, \ldots, g_{m}\right)$ converge almost everywhere to the product $g_{1} \cdots g_{m}$ as $R \rightarrow \infty$ whenever $g_{j} \in L^{p_{j}}(\mathbb{T})$. The previous analysis reduces this problem to the $L^{p_{1}} \times \cdots \times L^{p_{m}} \rightarrow L^{p}$ boundedness of the maximal operator defined on $\mathbb{R} \times \cdots \times \mathbb{R}$

$$
\mathscr{T}_{*}^{m}\left(f_{1}, \ldots, f_{m}\right)(x)=\sup _{N>0}\left|\int_{\left(\xi_{1} / N \ldots, \xi_{m} / N\right) \in E} \cdots \int_{f_{1}} \hat{f}_{1}\left(\xi_{1}\right) \cdots \hat{f}_{m}\left(\xi_{m}\right) e^{2 \pi i x\left(\xi_{1}+\cdots+\xi_{m}\right)} \mathrm{d} \xi_{1} \cdots \mathrm{d} \xi_{m}\right|
$$

which is a variant of a multilinear Carleson type operator. In the forthcoming publication, Muscalu, Thiele, and Tao (see [16] for the Walsh case) show that the following so-called bi-Carleson operator

$$
\mathscr{C}_{*}\left(f_{1}, f_{2}\right)(x)=\sup _{N>0}\left|\iint_{\xi_{1}<\xi_{2}<N} \hat{f}_{1}\left(\xi_{1}\right) \hat{f}_{2}\left(\xi_{2}\right) e^{2 \pi i x\left(\xi_{1}+\xi_{2}\right)} \mathrm{d} \xi_{1} \mathrm{~d} \xi_{2}\right|
$$

maps $L^{p_{1}}(\mathbb{R}) \times L^{p_{2}}(\mathbb{R})$ into $L^{p}(\mathbb{R})$ for all $1<p_{1}, p_{2} \leq \infty$ with $2 / 3<p<\infty$ whenever $1 / p_{1}+1 / p_{2}=1 / p$. Let us introduce a bilinear multiplier operator $S_{b}$ on $\mathbb{T} \times \mathbb{T}$ by setting

$$
S_{b}\left(g_{1}, g_{2}\right)(x)=\sum_{n_{1}<n_{2}} \hat{g}_{1}\left(n_{1}\right) \hat{g}_{2}\left(n_{2}\right) e^{2 \pi i x\left(n_{1}+n_{2}\right)}
$$


for all $g_{1}, g_{2}$ smooth functions on $\mathrm{T}$. The operator $S_{b}$ is a version of a discrete bilinear Hilbert transform and admits a bounded extension (also denoted by $S_{b}$ ) from $L^{p_{1}}(\mathbb{T}) \times L^{p_{2}}(\mathbb{T})$ to $L^{p}(\mathbb{T})$ via bilinear transference; see Fan and Sato [6] when $1<p_{1}, p_{2} \leq \infty, 2 / 3<p<\infty$, and $1 / p_{1}+1 / p_{2}=1 / p$.

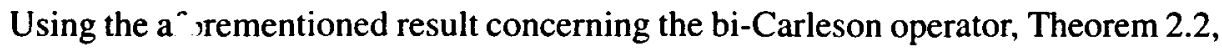
and Theorem $\lrcorner .2$ we deduce the following:

THEOREM 4.4. Let $1<p_{1}, p_{2}<\infty$ with $2 / 3<p<\infty$ whenever $1 / p_{1}+1 / p_{2}=$ $1 / p$. Then for $g_{j} \in L^{p_{j}}(\mathbb{T})$ we have

$$
\sum_{n_{1}<n_{2}<R} \hat{g}_{1}\left(n_{1}\right) \hat{g}_{2}\left(n_{2}\right) e^{2 \pi i x\left(n_{1}+n_{2}\right)} \rightarrow S_{b}\left(g_{1}, g_{2}\right)(x)
$$

as $R \rightarrow \infty$ for almost all $x$ in $\mathbb{T}$.

For our next application, we let again $D$ be a polygon in $\mathbb{R}^{2}$ containing 0 in its interior with no side parallel to the antidiagonal $y=-x$. We are interested in showing that the operators $P_{R}^{D}\left(g_{1}, g_{2}\right)$ converge a.e. to the product $g_{1} g_{2}$ whenever $g_{1}$ and $g_{2}$ are $L^{p_{j}}$ functions on the circle.

We can reduce the boundedness of the operator $T_{*}^{2}$ to that of $C_{*}$ in the following way. We first write $D$ as a union of at most four polygons each contained in one of the four quadrants and without loss of generality we may work with the part of $D$ in the first quadrant. Using Lemma 4.1 we can break $D$ as a difference of two finite unions of triangles with two sides parallel to the axes. Applying translations in Fourier space (modulations in time space), we may assume that all of the triangles that appear in the decomposition have an acute angle at the origin. This way we can pointwise control $T_{*}^{2}$ by a finite sum of operators of the form (for some $c>0$ )

$$
\mathscr{C}_{*}\left(f_{1}, f_{2}\right)(x)=\sup _{N>0}\left|\iint_{0<\xi_{1}<c \xi_{2}<N} \hat{f}_{1}\left(\xi_{1}\right) \hat{f}_{2}\left(\xi_{2}\right) e^{2 \pi i x\left(\xi_{1}+\xi_{2}\right)} \mathrm{d} \xi_{1} \mathrm{~d} \xi_{2}\right| .
$$

Using the boundedness of this version of the bi-Carleson operator, Theorem 2.2, and Theorem 3.2 we obtain the following:

THEOREM 4.5. Let $D$ be a polygon in $\mathbb{R}^{2}$ with no side parallel to the antidiagonal $y=-x$ that contains 0 in its interior. Let $1<p_{1}, p_{2} \leq \infty$ with $2 / 3<p<\infty$ whenever $1 / p_{1}+1 / p_{2}=1 / p$. Then for $g_{j} \in L^{p_{i}}(\mathbb{J})$ we have $P_{R}^{D}\left(g_{1}, g_{2}\right) \rightarrow g_{1} g_{2}$ almost everywhere on T as $R \rightarrow \infty$. If $D$ has a side parallel to the the antidiagonal $y=-x$, then the same conclusion is valid whenever $p>1$.

The preceding result may be viewed as a bilinear analogue of the Carleson-Hunt theorem $[3,10]$ on the almost every where convergence of Fourier series of $L^{p}$ functions on the circle (with respect to polygonal summation). 
Another analogue of the Carleson-Hunt theorem can be obtained using recent results by $\mathrm{Li}$ and Muscalu [14] who showed that the maximal operator obtained by considering the supremum of all the shifts of a Coifman-Meyer multiplier $\sigma$ on $\mathbb{R}^{m}$ is $L^{p}(\mathbb{R})$ bounded. (Taking $\sigma(\xi)=\chi_{(0, \infty)}$ when $m=1$, yields the Carleson-Hunt theorem.) The Coifman-Meyer symbols are those satisfying

$$
\left|\partial_{1}^{\alpha_{1}} \cdots \partial_{m}^{\alpha_{m}} \sigma\left(\xi_{1}, \ldots, \xi_{m}\right)\right| \leq C_{\alpha_{1}, \ldots, \alpha_{m}}\left(\left|\xi_{1}\right|+\cdots+\left|\xi_{m}\right|\right)^{-\left(\left|\alpha_{1}\right|+\cdots+\left|\alpha_{m}\right|\right)}
$$

for all sufficiently large multi-indices $\alpha_{1}, \ldots, \alpha_{m}$. The associated maximal operator is defined as the supremum of the operators $\left|T_{\sigma_{z}}\right|$ over all $z \in\left(\mathbb{R}^{n}\right)^{m}$, where $\sigma_{z}=$ $\sigma(\cdot+z)$. The result of [14] then says that this maximal operator is bounded from $L^{p_{1}}(\mathbb{R}) \times \cdots \times L^{p_{m}}(\mathbb{B})$ to $L^{p}(\mathbb{R})$ for any $p_{j}$ satisfying (2) with $1 / m<p<\infty$. Combining this theorem with Theorem 3.2 and Theorem 2.2 yields the following result:

THEOREM 4.6. Let $\sigma$ be a Coifman-Meyer multilinear multiplier on $\mathbb{R}^{m}$ which is continuous at zero and let $1<p_{j}<\infty, 1 / m<p<\infty$ be such that (2) holds. Then

$$
\lim _{z \rightarrow 0} T_{\sigma(++z)}\left(f_{1}, \ldots, f_{m}\right)=T_{\sigma}\left(f_{1}, \ldots, f_{m}\right) \text { a.e. }
$$

and

$$
\lim _{z \rightarrow 0} S_{\sigma(\cdot+z)}\left(g_{1}, \ldots, g_{m}\right)=S_{\sigma}\left(g_{1}, \ldots, g_{m}\right) \text { a.e. }
$$

for any $f_{j} \in L^{p_{j}}\left(\mathbb{R}^{n}\right)$ and $g_{j} \in L^{p_{j}}\left(\mathbb{T}^{n}\right)$.

\section{References}

[1] O. Blasco, 'Bilinear multipliers and transference', Int. J. Math. Math. Sci. 2005, 545-554.

[2] O. Blasco and F. Villaroya, 'Transference of bilinear multiplier operators on Lorentz spaces', Illinois J. Math. 47 (2003), 1327-1343.

[3] L. Carleson, 'On convergence and growth of partial sums of Fourier series', Acta Math. 116 (1966), 135-157.

[4] R. R. Coifman and G. Weiss, Transference methods in analysis, Conference Board of the Mathematical Sciences Regional Conference Series in Math. 31 (Amer. Math. Soc., Providence, RI, 1976).

[5] K. de Leeuw, 'On $L_{p}$ multipliers', Ann. of Math. (2) 81 (1965), 364-379.

[6] D. Fan and S. Sato, 'Transference of certain multilinear multiplier operators', J. Austral. Math. Soc. 70 (2001), 37-55.

[7] L. Grafakos, Classical and modern Fourier analysis (Prentice Hall, Upper Saddle River NJ, 2004).

[8] L. Grafakos and X. Li, 'The disc as a bilinear multiplier', preprint.

[9] L. Grafakos and G. Weiss, 'Transference of multilinear operators', Illinois J. Math. 40 (1996), 344-351. 
[10] R. Hunt, 'On the convergence of Fourier series', in: Orthogonal Expansions and Their Continuous Analogues (Edwardsville, IL, 1967) (ed. D. T. Haimo) (Southern Illinois Univ. Press, Carbondale IL, 1968) pp. 235-255.

[11] C. Kenig and P. Tomas, 'Maximal operators defined by Fourier multipliers', Studia Math. 68 (1980), 79-83.

[12] M. Lacey and C. Thiele, ' $L$ ' bounds for the bilinear Hilbert transform, $p>2$ ', Ann. of Math. (2) 146 (1997), 693-724.

[13] - 'On Calderón's conjecture', Ann. of Math. (2) 149 (1999), 475-496.

[14] X. Li and C. Muscalu, 'Generalizations of the Carleson-Hunt theorem. I. The classical singularity case', preprint.

[15] M. Murray, 'Multilinear convolutions and transference', Michigan Math. J. 31 (1984), 321-330.

[16] C. Muscalu, C. Thiele and T. Tao, 'A discrete model for the bi-Carleson operator', Geom. Func. Anal. 12 (2002), 1324-1364.

Department of Mathematics

University of Missouri

Columbia MO 65211

USA

e-mail: loukas@math.missouri.edu
Department of Mathematics

Michigan State University

East Lansing MI 48824

USA

e-mail: petrhonz@math.msu.edu 